\title{
Adherence to Lifelines Diet Score (LLDS) Is Associated with a Reduced Risk of Breast Cancer (BrCa): A Case-Control Study
}

\author{
Mohammad Hassan Sohouli $\left(D,{ }^{1,2}\right.$ Mohammad Hadizadeh ${ }^{(D},{ }^{1}$ Morteza Omrani $(D){ }^{3}$ \\ Mansoureh Baniasadi $\left({ }_{0},{ }^{1}\right.$ Vahid Sanati $(\mathbb{1})^{3}$ and Mitra Zarrati ${ }^{3}{ }^{3}$ \\ ${ }^{1}$ Cancer Research Center, Shahid Beheshti University of Medical Sciences, Tehran, Iran \\ ${ }^{2}$ Department of Clinical Nutrition and Dietetics, Faculty of Nutrition and Food Technology, \\ Shahid Beheshti University of Medical Sciences, Tehran, Iran \\ ${ }^{3}$ Department of Nutrition, School of Public Health, Iran University of Medical Sciences, Tehran, Iran \\ Correspondence should be addressed to Mitra Zarrati; khanomiana@yahoo.com \\ Mohammad Hassan Sohouli and Mohammad Hadizadeh contributed equally to this work.
}

Received 30 September 2021; Revised 20 November 2021; Accepted 14 December 2021; Published 4 February 2022

Academic Editor: Bing Niu

Copyright (c) 2022 Mohammad Hassan Sohouli et al. This is an open access article distributed under the Creative Commons Attribution License, which permits unrestricted use, distribution, and reproduction in any medium, provided the original work is properly cited.

\begin{abstract}
Background. Previous evidence suggests a link between diet quality and breast cancer (BrCa); however, the link between the Lifelines Diet Score (LLDS) - a fully food-based score that uses the 2015 Dutch Dietary Guidelines - and risk of BrCa has not yet been evaluated. Therefore, the aim of this study was to observe the relationship between adherence to an LLDS and risk of BrCa in Iranian adults. Methods. In the hospital-based case-control study, 253 patients with $\mathrm{BrCa}$ and 267 non-BrCa controls were enrolled. Individual's food consumption was recorded to calculate LLDS using a semiquantitative food frequency questionnaire. In adjusted models, the association between the inflammatory potential of the diet and the risk of BrCa was estimated by using binary logistic regression. Results. Compared with control individuals, $\mathrm{BrCa}$ patients significantly had higher waist circumference (WC), first pregnancy age, abortion history, and number of children. In addition, the mean intake of vitamin $D$ supplements and anti-inflammatory drugs in the case group was significantly lower than the control group. Furthermore, after adjusted potential confounders, individuals in the highest vs. lowest quartiles of LLDS showed statistically significant lower risk of BrCa in overall population (OR: 0.21; 95\% CI: 0.11-0.43; P trend <0.001), premenopausal (OR: 0.26; 95\% CI: 0.10-0.68; P trend $=0.003$ ), and postmenopausal women (OR: 0.20; 95\% CI: 0.06-0.60; P trend =0.015). Conclusion. Findings of this study reflected that higher LLDS decreased risk of $\mathrm{BrCa}$, but need further investigation in later studies.
\end{abstract}

\section{Introduction}

Breast cancer $(\mathrm{BrCa})$ is one of the most common cancers in women that rapidly increasing worldwide [1]. One in eight women in Western societies is reported to suffer from this disease, and this amount is about 22.6 per 100,000 females, among Iranian population [2, 3]. There are two types BrCa risk factors which include intrinsic factors like genetic variations that plays an important role in $\mathrm{BrCa}$ incidence and extrinsic factors causing cancer, like lifestyle-related factors such as low physical activity and carcinogenic dietary habits. Meanwhile, the role of unhealthy food patterns such as high-saturated fat and high-sugar diets, and consumption of more high-fat dairy products in breast cancer has been more prominent $[4,5]$. Past studies have also found that modifying the individual diet with more intake of fruit and vegetables and reducing the consumption of sweets are one of the best ways to prevent breast cancer [5-10]. But due to the comprehensiveness of dietary patterns and their more reliable association with various chronic diseases, several nutritional indices have emerged. These indices include the Mediterranean Diet Scores (MED) score and the Healthy Eating Index (HEI). One of the main components of these two scores is to consider more fruits and vegetables in the 
diet, and relationship between these indices and reduced the risk of various diseases such as cancer has been shown [11-15]. Although both scores consider consuming saturated and unsaturated fatty acids, but in the Mediterranean diet, it is recommended to reduce dairy consumption, while previous studies have shown that dairy consumption such as yogurt is inversely related to some cancer risk such as colon and breast cancer [16-18]. Furthermore, in the Mediterranean score, there is not a recommendation about sweetened drinks with diet sugar, the carcinogenic effects of which have been proven in breast cancer [19]. Due to deficiencies such as the fact that explain these two scores are not totally dietbased, a new score called Lifelines Diet Score (LLDS) has emerged. Lifelines Diet Score (LLDS) is a brand new score based on food, which is according to the Dutch Dietary Guidelines, in order to find the association of diseases with diets [17]. In this score, food groups were classified according to LLDS guidelines as foods with positive (vegetables, fruits, whole grain products, legumes and nuts, fish, oil and soft margarine, unsweetened dairy products, coffee, and tea), negative (red and processed meat, butter and hard margarine, and sugar-sweetened beverages), neutral, or unknown effects on health, in which neutral and unknown food groups are not involved in the LLDS calculation [17]. According to our information, so far, no study has been conducted to investigate the effect of LLDS with risk of cancer, especially breast cancer; however, two studies examine the relationship between this score and sleep quality in obese individuals and another study conducted in order to find the relationship between LLDS and type 2 diabetes incidence in the Dutch Lifelines cohort $[20,21]$. The results of the two studies showed that higher scores improve sleep quality in obese individuals and reduce the risk of type 2 diabetes in the Dutch Lifelines cohort, respectively [20, 21]. We hypothesized that people who consumed a diet high in fruits and vegetables, as well as eating less red meat and sugary drinks, had a higher LLDS and reduced levels of cancer-causing agents in the body; as a result, the risk of $\mathrm{BrCa}$ can be reduced. Thus, the aim of the present study was to investigate the association between LLDS and risk of $\mathrm{BrCa}$ in a case-control study.

\section{Methods}

The type of study is a case-control study. The target population of this study was 520 samples (two hundred and fiftythree people diagnosed with $\mathrm{BrCa}$ and two hundred and sixty-seven people without the disease). It should be noted that this number of samples were selected from "Hazrat Rasoul and Taleghani Hospital, Tehran, Iran," in a period of one year between the years 2019 and 2020. The minimum required sample size was calculated based on the ability to detect an OR of 2 with a case to control ratio of $1: 1,90 \%$ power, and a type I error $(\alpha)$ rate of $5 \%$ [22].

BrCa patients were newly ( $<6$ months) diagnosed with histologically confirmed $\mathrm{BrCa}$ by an oncologist. Patients with a history of other cancers, hormone-related diseases such as PCOS (polycystic ovary syndrome) or endometriosis, and occurrence of metastasis were also excluded from our study. The control group was selected from other parts of the hospital such as eye; ear, nose, and throat; skin; and beauty; did not have any history of cancer (benign and malignant), inflammatory disease, and hormone-related diseases; and for the past 6 months, they have had a regular diet. Matching between two groups was done based on age and BMI. The control group was selected from other departments of the hospital, such as ophthalmology, otolaryngology, dermatology, and aesthetics. The group did not have any history of cancer (benign and malignant), inflammatory disease, and hormone-related diseases. Also, each member of the control group included in the study was examined for inflammation by an internal medicine specialist and laboratory results to ensure the absence of inflammation. In order to determine the level of physical activity of individuals, a valid short form of the International Physical Activity Questionnaire (short IPAQ) was used, and informed written consent was obtained from all patients. Finally, it is important to note that this study was accepted via the Research Council and the Ethics Committee of Iran University of Medical Sciences, Tehran, Iran.

2.1. Dietary Assessment. A valid questionnaire of semiquantitative food frequency (including 168 food items) was used to determine food consumption compared to last year [23]. The main feature of this FFQ was to follow the usual Iranian food with standard serving size, and for each food, the regular amount of portion eaten and the number of times consumed by the participant was obtained by filling in the FFQ. Consumption for each food consisted as follows: at no time, two-three times/month, one time/week, two-four times/week, five-six times/week, and every day. Portion sizes were transformed to gram, by typical Iranian family measures [24]. Day-to-day nutrient consumptions for each individual were estimated by use of the United States Department of Agriculture's (USDA) national nutrient databank [25]. Nutritionist IV software was applied to determine the everyday energy and nutrients consumption for each subject.

2.2. Assessment of Nondietary Exposures. A skilled questioner managed all other questionnaires. So, all the attendee answered properly to the study queries. Overall appearances and medical data gathered through FFQ, consisted of age (years), age at 1st pregnancy (years), menopausal status (premenopause or postmenopause), literacy status, oral contraceptive medicines intake history (month), history of benign breast illness (yes or no), family history of cancer (yes or no), BrCa household history (yes or no), bra wearing at night (yes or no), smoking (yes or no), supplement consumption (yes or no; if yes, the corresponding info about dosage and intake times), NSAIDs usage (yes or no), and contact to sunlight throughout the day ((1) less than half an hour a day, (2) between half an hour and one hour a day, (3) between one and two hours a day, (4) more than two hours a day). Anthropometric quantities were gotten by means of standardized procedures. Weight was measured while patients wearing comfortable clothes, barefooted by a digital 
Seca scale (made in Germany) with the precision of one hundred grams. Height was obtained in a standing position barefooted, by means of a tape meter. Body mass index (BMI) was considered as weight $(\mathrm{kg})$ divided by the square of height (m2). Waist circumference (WC) was obtained by means of nonelastic tap at the slimmest level deprived of any heaviness to body surface.

2.3. Calculation of Lifelines Diet Score. LLDS was computed originated from the process described in the study by Vinke et al. [17]. In this score, food groups were classified according to LLDS guidelines as foods with positive, negative, neutral, or unknown effects on health, which neutral (eggs) and unknown food (included potatoes, refined grain products, white unprocessed meat, cheese, ready-to-eat savory products, sugary products, soups, sweetened dairy, and artificially sweetened products) groups are not involved in the LLDS calculation. Nine foods including vegetables, fruits, whole grain products, legumes and nuts, fish, oil and soft margarine, unsweetened dairy products, coffee, and tea were considered as foods with positive effects. In addition, foods with negative effects include 3 food groups: red and processed meat, butter and hard margarine, and sugar-sweetened beverages. In order for LLDS to show the quality of the relative diet, taking into account the difference in energy consumption between individuals, food intake was calculated in terms of grams per $1000 \mathrm{kcal}(\mathrm{kcal})$ instead of grams per day for each food group and the individual's results divided into quintiles from one to five, with "one" scores the minimum consumption in each food group and "five" scores the maximum. Summing the scores of the twelve components leads to an LLDS score of twelve to sixty.

2.4. Statistical Analyses. Statistical analysis was performed using SPSS 20 software (version 19.0; SPSS Inc., Chicago IL). The normalcy of variables was considered by Shapiro-Wilk exams. Mean values of more than 2 groups were evaluated by means of a nalysis of variance (ANOVA) for normal distribution variables. Furthermore, the chi-square test was intended for comparing categorical variables. The risk of breast cancer (OR) and respective 95\% confidence interval $(95 \% \mathrm{CI})$ were estimated by binary logistic regression analysis with adjustment for confounders. In order to calculate ORs and 95\% CIs, we classified all subjects based on the LLDS score into quartile ranges using the rank cases and then entered into the logistic regression as a categorical variable. The overall Ptrend across increasing quartiles was examined by considering the median score in each category as a continuous variable. We adjusted the results in three models using a priori selected potential confounders, which included model 1-age and BMI, model 2-additional adjustment for waist circumference, energy, first pregnancy age, number of children, history of abortion, hormone replacement therapy, use of anti-inflammatory drugs, and vitamin D supplementation. The data were presented as mean \pm standard deviation, and statistical significance was accepted, a priori, at $P<0.05$.
2.5. Result. The mean \pm SD for the age and BMI of the study population was $47.92 \pm 10.33$ years and $29.43 \pm 5.51 \mathrm{~kg} . \mathrm{m} 2$, respectively.

Demographic characteristics, lifestyle, and medical history of study participants among case and control groups as well as across quartiles of LLDS are shown in Table 1. Compared with control individuals, $\mathrm{BrCa}$ patients significantly had higher waist circumference (WC), first pregnancy age, abortion history, and number of children. In addition, the mean intake of vitamin $\mathrm{D}$ supplements, hormone replacement therapy, and anti-inflammatory drugs in the case group was significantly lower than the control group. However, no significant differences were found for other characteristics and variables among cases and controls subjects as well as across quartiles of LLDS.

The mean dietary intake of the participants in the study based on case and control groups as well as quartiles of LLDS is shown in Table 2. Although subjects with $\mathrm{BrCa}$ had higher intake of energy, fat, saturated fatty acids (SFA), cholesterol, carbohydrates, sodium, folate, and iron than controls, but they had lower intake of mono- and polyunsaturated fatty acids (MUFA and PUFA), potassium, phosphorus, calcium, vitamin B12, and micronutrients antioxidants such as zinc, magnesium, and vitamins $\mathrm{E}, \mathrm{C}$, and $\mathrm{D}$. Also, the intake of protein, potassium, phosphorus, calcium, magnesium, zinc, vitamins $C$, and $D$ in the highest quartiles of LLDS vs. the lowest quartiles of LLDS significantly increased; however, the intake of fats, MUFA, and PUFA decreased.

Table 3 shows the dietary intake of 12 components LLDS (in grams per $1000 \mathrm{kcal}$ ) among study subjects based on the case and control groups as well as quartiles this score. Among the positive components LLDS, BrCa patients significantly had lower consumption of healthy food groups including vegetables, fruits, and legumes and nuts and higher intake of coffee. Furthermore, among the negative components of this score, red and processed meat and sugarsweetened beverages intake were significantly higher in the case group than the control group. Across quartiles of LLDS, all the positive components of this score except coffee and tea significantly increased and its negative components significantly decreased.

The Odds ratios (ORs) and 95\% confidence intervals (CIs) for BrCa patients according to quartiles of LLDS gives in Table 4. In crude and adjusted model 1(age and BMI adjusted), we observed a significant decreased odds ratio of $\mathrm{BrCa}$ across quartiles of LLDS in the overall population of women (OR: 0.31; 95\% CI: 0.17-0.56; P trend $<0.001$ and OR: $0.28,95 \%$ CI: $0.15-0.52$; $\mathrm{P}$ trend $<0.001)$, premenopausal (OR: 0.35 ; 95\% CI: $0.16-0.77$; $\mathrm{P}$ trend $=0.003$ and OR: 0.38 , 95\% CI: $0.10-0.68$; $\mathrm{P}$ trend $=0.003)$, and post-menopausal women(OR: 0.23 ; 95\% CI: $0.08-0.63$; P trend $=0.017$ and OR: 0.22 , 95\% CI: $0.08-0.63$; P trend $=0.020$ ), respectively. Furthermore, after additional adjusting for WC, energy, first pregnancy age, number of children, history of abortion, use of anti-inflammatory drugs, and vitamin $D$ supplementation in the finally adjusted model, individuals in the highest vs lowest quartiles of LLDS showed statistically significant lower risk of $\mathrm{BrCa}$ in all three categories (OR: 0.21 ; 95\% CI: $0.11-0.43$; $\mathrm{P}$ trend $<0.001$ for overall 
TABLE 1: Demographic, anthropometric, and lifestyle characteristics of participants across case and control groups as well as quartiles of LLDS.

\begin{tabular}{|c|c|c|c|c|c|c|}
\hline \multirow[t]{2}{*}{ Variables } & \multicolumn{2}{|c|}{ Groups, mean (SD) } & \multicolumn{3}{|c|}{$\begin{array}{l}\text { Quartiles of LLDS, mean } \\
\text { (SD) }\end{array}$} & \multirow[t]{2}{*}{$P$ value } \\
\hline & Case $(n=253)$ & Control $(n=267)$ & & Q1 & $\mathrm{Q} 4$ & \\
\hline Age, y & $48.91(10.46)$ & $47.13(10.08)$ & 0.062 & $48.21(10.28)$ & $48.86(10.67)$ & 0.957 \\
\hline $\mathrm{BMI}^{\mathrm{b}}, \mathrm{kg} / \mathrm{m}^{2}$ & $29.61(4.55)$ & $29.07(5.39)$ & 0.222 & $29.19(4.88)$ & $29.50(5.10)$ & 0.970 \\
\hline Waist- circumference $(\mathrm{cm})$ & $101.15(96.39)$ & $96.39(13.25)$ & $<0.001$ & $98.34(10.70)$ & $97.89(12.68)$ & 0.439 \\
\hline Physical activity (Met.h/wk) & $33.18(6.11)$ & $32.70(5.20)$ & 0.336 & $32.53(4.88)$ & $33.12(5.89)$ & 0.513 \\
\hline Smoking (yes), $n(\%)$ & (3.2) 8 & (3.4) 9 & 0.894 & $3(2.5)$ & $3(2.5)$ & 0.690 \\
\hline Marriage age, y & $19.43(5.02)$ & $18.98(4.48)$ & 0.296 & $19.28(5.22)$ & $19.00(4.14)$ & 0.939 \\
\hline First pregnancy age, y & $22.29(5.32)$ & $20.35(4.19)$ & $<0.001$ & $21.92(5.04)$ & $20.88(4.89)$ & 0.334 \\
\hline Child number & $2.92(1.43)$ & $2.54(1.59)$ & 0.005 & $2.73(1.46)$ & $2.79(1.54)$ & 0.782 \\
\hline Abortion history (yes), $n(\%)$ & $(37.2) 94$ & $(29.2) 78$ & 0.049 & $40(33.6)$ & $43(35.2)$ & 0.927 \\
\hline Menopausal status (post-menopausal), $n(\%)$ & $115(45.5)$ & $114(42.7)$ & 0.527 & $48(21)$ & $59(25.8)$ & 0.652 \\
\hline Hormone replacement therapy (yes), $n(\%)$ & $13(5.1)$ & $25(10.9)$ & 0.016 & $4(9.5)$ & $13(31)$ & \\
\hline Family history of breast cancer (yes), $n$ (\%) & $(5.5) 14$ & $(4.5) 12$ & 0.594 & $6(5.0)$ & $9(7.4)$ & 0.479 \\
\hline Family history of cancer (yes), n (\%) & $(26.9) 68$ & (20.7) 55 & 0.097 & $27(22.7)$ & $29(23.8)$ & 0.801 \\
\hline Benign breast diseases history (yes), $n(\%)$ & (7.9) 20 & $(5.3) 14$ & 0.224 & $9(7.6)$ & $6(4.9)$ & 0.712 \\
\hline Inflammatory disease history (yes), $n(\%)$ & (12.6) 32 & (13.2) 35 & 0.863 & $12(10.1)$ & $17(13.9)$ & 0.068 \\
\hline Vitamin D supplement (yes), $n(\%)$ & $(14.6) 37$ & $(24.3) 65$ & 0.005 & $23(19.3)$ & $21(17.2)$ & 0.177 \\
\hline Ever use of OCP (yes), $n(\%)$ & (49.8) 126 & (56) 149 & 0.156 & $54(45.4)$ & $67(54.9)$ & 0.253 \\
\hline Anti-inflammatory drugs use (yes), $n(\%)$ & $(10.3) 26$ & (17.7) 47 & 0.015 & $19(16.0)$ & $16(13.1)$ & 0.525 \\
\hline Education status, $n(\%)$ & & & 0.518 & & & 0.143 \\
\hline Illiterate & $(11.6) 29$ & (9) 24 & & $19(16.1)$ & $6(4.9)$ & \\
\hline Low education & $116(46.6)$ & (50.4) 134 & & $54(45.8)$ & $65(53.3)$ & \\
\hline Higher education & (42) 105 & (40.6) 108 & & $45(38.1)$ & $51(41.8)$ & \\
\hline Exposure to sunlight during the day ( $\mathrm{min}$ ) & & & 0.215 & & & 0.398 \\
\hline Less than 30 minutes & $(28.5) 72$ & (36) 96 & & $30(25.2)$ & $44(36.1)$ & \\
\hline $60-30$ minutes & $(32.4) 82$ & $(25.5) 68$ & & $42(35.3)$ & $33(27.0)$ & \\
\hline $120-60$ minutes & (17) 43 & (17.2) 46 & & $19(16.0)$ & $15(12.3)$ & \\
\hline More than 120 minutes & (22.1) 56 & $(21.3) 57$ & & $28(23.5)$ & $30(24.6)$ & \\
\hline
\end{tabular}

${ }^{a}$ Obtained from ANOVA for continuous variables and Chi-square for aategorical variables. ${ }^{\mathrm{b}} \mathrm{BMI}$ : body mass index.

TABLE 2: Dietary intakes of study participants across case and control groups as well as quartiles of LLDS.

\begin{tabular}{|c|c|c|c|c|c|c|}
\hline & \multicolumn{2}{|c|}{ Groups, mean $(\mathrm{SD})$} & \multirow{2}{*}{$P$ value $^{\mathrm{a}}$} & \multicolumn{2}{|c|}{ Quartiles of LLDS, mean (SD) } & \multirow{2}{*}{$P$ value } \\
\hline & Case $(n=253)$ & Control $(n=267)$ & & Q1 & Q4 & \\
\hline Energy $(\mathrm{kcal} / \mathrm{d})$ & $2753.45(798.02)$ & $2464.1(607.43)$ & $<0.001$ & $2684.42(770.14)$ & $2592.07(669.86)$ & 0.589 \\
\hline Carbohydrate (g/d) & $372.54(7.01)$ & $343.54(5.49)$ & 0.001 & $357.55(102.14)$ & $362.94(97.10)$ & 0.859 \\
\hline Protein $(g / d)$ & $80.08(1.49)$ & $88.47(1.56)$ & $<0.001$ & $78.33(23.85)$ & $92.51(26.36)$ & $<0.001$ \\
\hline Fat $(g / d)$ & $108.24(2.55)$ & $91.82(2.20)$ & $<0.001$ & $110.10(48.52)$ & $92.50(28.38)$ & 0.006 \\
\hline SFA $(g / d)$ & $32.92(11.26)$ & $29.20(10.53)$ & $<0.001$ & $32.31(13.21)$ & $29.99(9.44)$ & 0.418 \\
\hline MUFA (g/d) & $32.29(13.29)$ & $37.24(15.97)$ & $<0.001$ & $33.25(16.54)$ & $24.92(13.36)$ & $<0.001$ \\
\hline PUFA (g/d) & $20.48(10.35)$ & $24.49(13.29)$ & $<0.001$ & $26.21(14.65)$ & $18.95(7.79)$ & $<0.001$ \\
\hline Cholesterol (mg/d) & $293.52(135.55)$ & $261.88(139.27)$ & 0.009 & $275.98(131.08)$ & $286.87(146.13)$ & 0.850 \\
\hline Fiber $(\mathrm{g} / \mathrm{d})$ & $37.96(19.28)$ & 39.89 (18.58) & 0.247 & $40.19(22.91)$ & $38.76(13.40)$ & 0.417 \\
\hline Sodium $(\mathrm{mg} / \mathrm{d})$ & $4740.74(1811.95)$ & $4307.06(1898.50)$ & 0.008 & $4847.81(2163.35)$ & $4265.17(1454.76)$ & 0.073 \\
\hline Potassium (mg/d) & $3766.23(1224.29)$ & $4297.22(1261.12)$ & $<0.001$ & $3484.51(1195.27)$ & $4725.62(1184.66)$ & $<0.001$ \\
\hline Phosphor (mg/d) & $1482.87(492.60)$ & $1617.48(485.35)$ & 0.002 & $1367.58(472.82)$ & $1745.82(509.14)$ & $<0.001$ \\
\hline Iron $(\mathrm{mg} / \mathrm{d})$ & $20.28(9.96)$ & $16.34(6.06)$ & $<0.001$ & $18.38(7.87)$ & $18.50(7.79)$ & 0.917 \\
\hline Calcium (mg/d) & $1215.79(463.90)$ & $1335.27(458.76)$ & 0.003 & $1084.30(452.25)$ & $1453.21(498.15)$ & $<0.001$ \\
\hline Magnesium (mg/d) & 370.06 (119.89) & $402.91(133.15)$ & 0.003 & 335.84 (106.98) & $442.72(119.72)$ & $<0.001$ \\
\hline $\operatorname{Zinc}(\mathrm{mg} / \mathrm{d})$ & $11.76(3.82)$ & $12.95(4.05)$ & 0.001 & $11.49(3.95)$ & $13.52(3.65)$ & $<0.001$ \\
\hline Vitamin C (mg/d) & $159.16(89.15)$ & $197.87(78.89)$ & $<0.001$ & $147.07(82.51)$ & $222.93(84.78)$ & $<0.001$ \\
\hline Folate $(\mathrm{mcg} / \mathrm{d})$ & $485.57(168.28)$ & $455.20(163.07)$ & 0.037 & $465.79(186.66)$ & $468.61(130.00)$ & 0.977 \\
\hline Vitamin B12 (mcg/d $)$ & $5.53(3.87)$ & $6.70(4.53)$ & 0.002 & $6.46(5.76)$ & $6.09(2.84)$ & 0.740 \\
\hline Vitamin E (mg/d) & $17.64(13.16)$ & $23.59(17.54)$ & $<0.001$ & $22.53(21.15)$ & $18.38(9.66)$ & 0.232 \\
\hline Vitamin D $(\mathrm{mcg} / \mathrm{d})$ & $2.04(3.44)$ & $2.7(3.06)$ & 0.012 & $1.46(1.87)$ & $2.41(3.27)$ & $<0.001$ \\
\hline
\end{tabular}

Abbreviations; SFA, saturated fatty acid. ${ }^{\text {a } O b t a i n e d ~ f r o m ~ A N O V A . ~}$ 
TABLE 3: Dietary consumption of the 12 components included in the Lifelines Diet Score (LLDS) in grams per 1000 kcal among participants study (base case and control groups) and quartiles of LLDS.

\begin{tabular}{|c|c|c|c|c|c|c|}
\hline & \multicolumn{2}{|c|}{ Groups, mean (SD) } & \multirow{2}{*}{$P$ value $^{\mathrm{a}}$} & \multicolumn{2}{|c|}{ Quartiles of LLDS, mean (SD) } & \multirow{2}{*}{$P$ value $^{\mathrm{a}}$} \\
\hline & Case $(n=253)$ & Control $(n=267)$ & & Q1 & Q4 & \\
\hline LLDS score & $34.72(5.85)$ & $37.42(5.32)$ & $<0.001$ & $28.66(2.19)$ & $43.87(2.78)$ & $<0.001$ \\
\hline \multicolumn{7}{|l|}{ Positive components } \\
\hline Vegetables & $113.60(55.70)$ & $128.86(48.96)$ & 0.001 & $89.47(37.69)$ & $159.81(51.43)$ & $<0.001$ \\
\hline Fruits & $168.43(85.70)$ & $187.44(67.71)$ & 0.005 & $140.70(76.29)$ & $225.03(77.74)$ & $<0.001$ \\
\hline Whole grain products & $36.07(29.73)$ & $33.32(30.08)$ & 0.295 & $24.04(21.60)$ & $41.82(26.12)$ & $<0.001$ \\
\hline Legumes and nuts & $12.55(10.20)$ & $16.63(9.66)$ & $<0.001$ & $11.59(10.30)$ & $19.15(9.85)$ & $<0.001$ \\
\hline Fish & $4.50(5.07)$ & $5.39(5.52)$ & 0.058 & $3.25(3.86)$ & $7.10(7.05)$ & $<0.001$ \\
\hline Oils and soft margarines & $0.30(0.72)$ & $0.38(0.73)$ & 0.206 & $0.04(0.11)$ & $0.76(1.05)$ & $<0.001$ \\
\hline Unsweetened dairy & $194.18(106.59)$ & $197.69(116.36)$ & 0.721 & $147.86(87.44)$ & $242.81(117.06)$ & $<0.001$ \\
\hline Coffee & $0.02(0.08)$ & $0.00(0.02)$ & 0.008 & $0.00(0.02)$ & $0.02(0.06)$ & 0.211 \\
\hline Tea & $1.23(0.94)$ & $1.21(0.79)$ & 0.845 & $1.16(1.00)$ & $1.26(0.76)$ & 0.784 \\
\hline \multicolumn{7}{|l|}{ Negative components } \\
\hline Red and processed meat & $12.98(8.86)$ & $11.01(6.49)$ & 0.004 & $15.91(10.00)$ & $9.81(6.19)$ & $<0.001$ \\
\hline Butter, hard margarines & $14.26(8.42)$ & $13.92(8.76)$ & 0.659 & $18.59(9.50)$ & $9.44(5.73)$ & $<0.001$ \\
\hline Sugar-sweetened beverages & $33.24(37.08)$ & $22.63(23.57)$ & $<0.001$ & $45.55(43.33)$ & $16.18(11.63)$ & $<0.001$ \\
\hline
\end{tabular}

${ }^{a}$ Obtained from ANOVA.

TABLE 4: Odds ratio (OR) and 95\% confidence interval (CI) for breast cancer based on quartiles of food-based Lifelines Diet Score (LLDS).

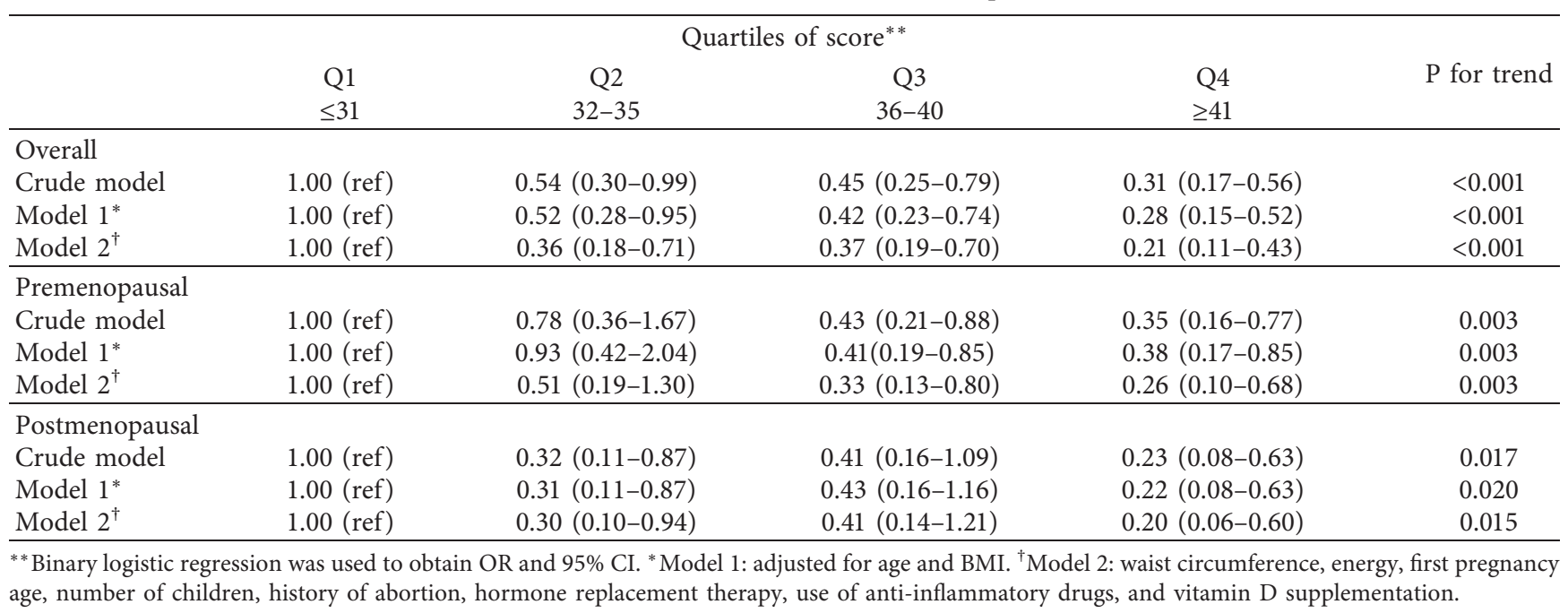

population, OR: 0.26 ; 95\% CI: $0.10-0.68 ; \mathrm{P}$ trend $=0.003$ for premenopausal, and OR: $0.20 ; 95 \% \mathrm{CI}: 0.06-0.60 ; \mathrm{P}$ trend $=0.015$ for post-menopausal women).

\section{Discussion}

The aim of our study was to investigate the relationship between the adherence to the LLDS and risk of BrCa. The results showed that adherence to a diet with a higher LLDS, which is high in vegetables, fruits, and legumes and nuts can reduce risk of $\mathrm{BrCa}$ in in the overall population. Also, in the highest quartile compared to the lower quartile of LLDS, a 74 and $80 \%$ reduction in the risk of $\mathrm{BrCa}$ was observed in premenopausal and post-menopausal women, respectively. Which, this risk reduction was greater in post-menopausal women.

Although our study examined association of LLDS and $\mathrm{BrCa}$ for the first time, a similar study with the same root mechanism of action was performed in relation to LLDS and risk of type 2 diabetes by Vinke et al. The result of this study indicated that LLDS can decrease the risk of type 2 diabetes in the Dutch Lifelines cohort [21]. Khani et al. published a case-control study on 278 overweight and obese women in 2020 years, which showed higher LLDS associated with better sleep quality [20].

Previous studies have also shown an association between MED and HEI dietary pattern indices, which in most respects are similar to LLDA, with a reduced risk of chronic diseases such as BrCa. Although, due to the differences that mentioned above between these indices with LLDS, the results cannot be fully generalized to our work. However, a case-control study by Turati et al. in 2018 years in Italy and Switzerland, the results showed that adherence to the Mediterranean diet significantly reduced the risk of $\mathrm{BrCa}$ in pre- and post-menopausal women [14]. In another study, adherence to a Western diet with a high content of meat and 
processed foods, sweetened foods with sugar was associated with a higher risk of $\mathrm{BrCa}$ (OR for the top vs the bottom quartile 1.46 (95\% CI 1.06-2.01)), especially in premenopausal women $(\mathrm{OR}=1.75 ; 95 \%$ CI $1.14-2.67)$ [26]. In contrast, the rich dietary pattern of olive oil, fruits and vegetables as a Mediterranean pattern (OR for the top quartile $v$ s the bottom quartile 0.56 (95\% CI 0.40-0.79)) was associated with a lower risk of this disease [26]. A metaanalysis consisting of cohort studies also showed a beneficial effect of the Mediterranean diet on reducing the incidence of $\mathrm{BrCa}$ [27]. Furthermore, Shahril et al. [13] conducted a casecontrol study in 2013 to examine the association of HEI with $\mathrm{BrCa}$ risk in 764 patients (382 BrCa cases and 382 healthy women). They concluded that, like the LLDS, a higher score of HEI reduces the risk of $\mathrm{BrCa}$. The components in this study showed that individuals who eat more fruits and vegetables have a higher score and a lower risk of BrCa. Also in this study, in line with our study, the reduction in BrCa risk in postmenopausal women was significantly greater than in premenopausal women, which could be due to the effect of estrogen, which is significantly reduced at menopausal age. Studies have also shown the important role of estrogen in the risk of BrCa and changes in female fat profile $[13,28]$.

According to result of our study, BrCa patients significantly had lower consumption of healthy food groups including vegetables, fruits, and legumes and nuts and higher intake of coffee. In line with our study, recently in NHS Health (NHS, 1980-2012) and NHSII (1991-1991) study on 182,145 women with $\mathrm{BrCa}$, the results showed that consuming more fruits and vegetables, especially cruciferous and yellow/orange vegetables ( $>5.5$ vs. $\leq 2.5$ servings/day $\mathrm{HR}=0.89,95 \% \mathrm{CI}=0.83-0.96$; $p_{\text {trend }}=0.006$ ), was associated with a significant reduction in $\mathrm{BrCa}$ [29]. In addition, recent meta-analysis studies have shown beneficial effects of fruit and vegetable intake on reducing the risk, mortality rate, and disease recurrence in these patients [30, 31].

Similarly, recently in a cohort study by Sanchez et al. with an annual follow-up of 115,802 individuals and 101 new cases of breast cancer among postmenopausal women in 2020 years, more than 1 cup of coffee per day was associated with a lower incidence of breast cancer (HR 0.44; 95\% confidence interval: $0.21,0.92$ ) [32]. However, in a metaanalysis study with a total of 21 prospective studies for doseresponse, 13 prospective studies showed no significant relationship between coffee consumption and $\mathrm{BrCa}$ risk in the nonlinear model [33]. However, when the analysis was limited to postmenopausal women, consuming four cups of coffee daily was associated with a $10 \%$ reduction in the risk of $\mathrm{BrCa}$ during menopause [33]. In addition, in line with our study, no significant association was found between moderate to low fish intake (2 portions per week or less) and the risk of breast cancer. However, high fish consumption (more than 4 portions per week) in adolescents and middle-aged people was associated with a $30 \%$ reduction in breast cancer [34]. Therefore, it seems that since the positive components of LLDS are rich in antioxidant compounds [35], such as omega 3 unsaturated fatty acids [36], and polyphenols [37], anti-inflammatory, and antioxidant pathways may be activated by increasing the consumption of these components and reducing the risk of $\mathrm{BrCa}$ [38-40]. On the other hand, these components often reduce visceral fat and stored body fat, by reducing estrogen, they can have beneficial effects on reducing the risk of $\mathrm{BrCa}$.

Among the negative components of this score, red and processed meat and sugar-sweetened beverages intake were significantly higher in the case group than the control group as mentioned in our study. It is more commonly consumed in BrCa patients. Sugar-sweetened beverages in these diets also increase insulin secretion. Insulin increases the secretion of growth-promoting substances such as IGF-1 (insulin like growth factor-1) causes higher growth of tumor and cancer cells, thus increasing the risk of cancer [41]. The higher score of LLDS index seems to indicate that adherence to a healthier diet is associated with higher consumption of beneficial micronutrients, dietary antioxidants, fruits, vegetables, and phytochemicals (as one of the anti-inflammatory compounds). Therefore, a consequential reduction in the odds of cancer by controlling the proliferation and growth of cancer cells, as well as the balance between the body's oxidative and antioxidant system, may be evident [42]. In addition, in recent years, many studies have shown an association between intake of red and processed meat with an increased incidence of $\mathrm{BrCa}[43,44]$. Red and processed meat in the diet with a lower LLDS have higher saturated fatty acids, saturated fatty acids can increase some pro-inflammatory substances such as TNF- $\alpha$ in the body that causes inflammation and tissue damage, and the body's cells are prone to various cancers, including $\mathrm{BrCa}$ [45-47]. However, no direct study has been performed on $\mathrm{BrCa}$ patient with our subject.

The current study has several strengths. To the best of our knowledge, it is the first case-control study assessing the association between LLDS with risk of $\mathrm{BrCa}$, which skilled people were used to interview and collect food frequency questionnaires. Our sample size was sufficient, and we tried to eliminate the effect of confounders as far as possible, by adjusting wide range of variables, and a validated questionnaire has been used. However, despite the novelty of this study, there are some limitations that should be noted. Despite the possible confounders considered in this study when analyzing, some confounders may not have been considered. Although we found evidence of an association between LLDS and $\mathrm{BrCa}$, due to the retrospective design utilized in this study, we cannot prove causality of the observed associations; therefore, future prospective studies and RCTs need to confirm the veracity of this finding. In addition, data were collected using self-report modalities, which are known to be associated with over- or under-reporting. However, we sought to ameliorate this by using trained interviewers and robustly validated tools.

\section{Conclusion}

Adherence to a diet with a higher LLDS decreased the odds of $\mathrm{BrCa}$, which this risk reduction was greater in postmenopausal women. However, due to the mentioned limitations, other prospective studies in this area have been suggested to be able to measure communication more fully. 


\section{Data Availability}

Data are available upon request due to privacy/ethical restrictions.

\section{Consent}

All authors of this manuscript declare their consent for publication.

\section{Disclosure}

The lead author affirms that this manuscript is an honest, accurate, and transparent account of the study being reported. The reporting of this work is in compliance with high quality qualitative research methodology. The lead author affirms that no important aspects of the study have been omitted and that any discrepancies from the study as planned have been explained. This manuscript is also written according to the STROBE guidelines.

\section{Conflicts of Interest}

The authors declare that they have no conflicts of interest.

\section{Authors' Contributions}

Mohammad Hassan Sohouli and Mohammad Hadizadeh contributed equally to this work.

\section{References}

[1] J. Seely and T. Alhassan, "Screening for breast cancer in 2018-what should we be doing today?" Current Oncology, vol. 25, no. Suppl 1, p. S115, 2018.

[2] S. B. Jazayeri, S. Saadat, R. Ramezani, and A. Kaviani, "Incidence of primary breast cancer in Iran: ten-year national cancer registry data report," Cancer epidemiology, vol. 39, no. 4, pp. 519-527, 2015.

[3] Z. Tao, A. Shi, C. Lu, T. Song, Z. Zhang, and J. Zhao, "Breast cancer: epidemiology and etiology," Cell Biochemistry and Biophysics, vol. 72, no. 2, pp. 333-338, 2015.

[4] M. Kamińska, T. Ciszewski, K. Łopacka-Szatan, P. Miotła, and E. Starosławska, "Breast cancer risk factors," Przeglad menopauzalny = Menopause review, vol. 14, no. 3, pp. 196-202, 2015.

[5] R. Baena Ruiz and P. Salinas Hernández, "Diet and cancer: risk factors and epidemiological evidence," Maturitas, vol. 77, no. 3, pp. 202-208, 2014.

[6] K. Ferrini, F. Ghelfi, R. Mannucci, and L. Titta, "Lifestyle, nutrition and breast cancer: facts and presumptions for consideration," Ecancermedicalscience, vol. 9, p. 557, 2015.

[7] T. T. Fung, S. E. Chiuve, W. C. Willett, S. E. Hankinson, F. B. Hu, and M. D. Holmes, "Intake of specific fruits and vegetables in relation to risk of estrogen receptor-negative breast cancer among postmenopausal women," Breast Cancer Research and Treatment, vol. 138, no. 3, pp. 925-930, 2013.

[8] S. Sieri, P. Chiodini, C. Agnoli et al., "Dietary fat intake and development of specific breast cancer subtypes," Journal of the National Cancer Institute, vol. 106, no. 5, 2014.

[9] S. Sulaiman, M. R. Shahril, S. W. Wafa, S. H. Shaharudin, and S. N. A. S. Hussin, "Dietary carbohydrate, fiber and sugar and risk of breast cancer according to menopausal status in
Malaysia," Asian Pacific Journal of Cancer Prevention, vol. 15, no. 14, pp. 5959-5964, 2014.

[10] J. Zang, M. Shen, S. Du, T. Chen, and S. Zou, "The association between dairy intake and breast cancer in western and Asian populations: a systematic review and meta-analysis," Journal of breast cancer, vol. 18, no. 4, p. 313, 2015.

[11] A. Salvatore Benito, M. Á. Valero Zanuy, M. Alarza Cano et al., "Adherence to Mediterranean diet: a comparison of patients with head and neck cancer and healthy population," Endocrinología, Diabetes y Nutrición (English ed.), vol. 66, no. 7, pp. 417-424, 2019.

[12] K. Hebestreit, M. Yahiaoui-Doktor, C. Engel et al., "Validation of the German version of the mediterranean diet adherence screener (MEDAS) questionnaire," BMC Cancer, vol. 17, no. 1, pp. 341-410, 2017.

[13] M. R. Shahril, S. Sulaiman, S. H. Shaharudin, and S. N. Akmal, "Healthy eating index and breast cancer risk among Malaysian women," European Journal of Cancer Prevention, vol. 22, no. 4, pp. 342-347, 2013.

[14] F. Turati, G. Carioli, F. Bravi et al., "Mediterranean diet and breast cancer risk," Nutrients, vol. 10, no. 3, p. 326, 2018

[15] N. Mourouti, M. D. Kontogianni, C. Papavagelis et al., "Adherence to the Mediterranean diet is associated with lower likelihood of breast cancer: a case-control study," Nutrition and Cancer, vol. 66, no. 5, pp. 810-817, 2014.

[16] G. Perdigon, AdM. de LeBlanc, J. Valdez, and M. Rachid, "Role of yoghurt in the prevention of colon cancer," European Journal of Clinical Nutrition, vol. 56, no. 3, pp. S65-S8, 2002.

[17] P. C. Vinke, E. Corpeleijn, L. H. Dekker, D. R. Jacobs, G. Navis, and D. Kromhout, "Development of the food-based Lifelines diet score (LLDS) and its application in 129,369 Lifelines participants," European Journal of Clinical Nutrition, vol. 72, no. 8, pp. 1111-1119, 2018.

[18] L. Chen, M. Li, and H. Li, "Milk and yogurt intake and breast cancer risk: a meta-analysis," Medicine, vol. 98, no. 12, 2019.

[19] A. Romanos-Nanclares, E. Toledo, I. Gardeazabal, J. J. Jiménez-Moleón, M. A. Martínez-González, and A. Gea, "Sugar-sweetened beverage consumption and incidence of breast cancer: the Seguimiento Universidad de Navarra (SUN) Project," European Journal of Nutrition, vol. 58, no. 7, pp. 2875-2886, 2019.

[20] S. Khani-Juyabad, L. Setayesh, H. Tangestani, N. Ghodoosi, S. F. Sajjadi, and N. Badrooj, "Adherence to lifelines diet score (llds) is associated with better sleep quality in overweight and obese women," Eating and Weight Disorders-Studies on Anorexia, Bulimia and Obesity, vol. 26, pp. 1-8, 2020.

[21] P. C. Vinke, G. Navis, D. Kromhout, and E. Corpeleijn, "Socio-economic disparities in the association of diet quality and type 2 diabetes incidence in the Dutch Lifelines cohort," EClinicalMedicine, vol. 19, Article ID 100252, 2020.

[22] S. Rigi, A. Salari-Moghaddam, S. Benisi-Kohansal, L. Azadbakht, and A. Esmaillzadeh, "Dietary glycaemic index and glycaemic load in relation to risk of breast cancer," Public Health Nutrition, vol. 11, no. 1, pp. 1-9, 2021.

[23] A. Keshteli, A. Esmaillzadeh, S. Rajaie, G. Askari, C. FeinleBisset, and P. Adibi, "A dish-based semi-quantitative food frequency questionnaire for assessment of dietary intakes in epidemiologic studies in Iran: design and development," International Journal of Preventive Medicine, vol. 5, no. 1, pp. 29-36, 2014.

[24] M. Ghaffarpour, A. Houshiar-Rad, and H. Kianfar, The Manual For Household Measures, Cooking Yields Factors and Edible Portion of Foods, p. 213, Keshaverzi Press, Tehran, Iran, 1999. 
[25] S. A. Bowman, J. E. Friday, and M. Alanna, MyPyramid Equivalents Database, 2.0 for USDA Survey Foods, 2003-2004: Documentation and User Guide, 2008.

[26] A. Castelló, M. Pollán, B. Buijsse et al., "Spanish Mediterranean diet and other dietary patterns and breast cancer risk: case-control EpiGEICAM study," British Journal of Cancer, vol. 111, no. 7, pp. 1454-1462, 2014.

[27] P. A. van den Brandt and M. Schulpen, "Mediterranean diet adherence and risk of postmenopausal breast cancer: results of a cohort study and meta-analysis," International Journal of Cancer, vol. 140, no. 10, pp. 2220-2231, 2017.

[28] L. Hilakivi-Clarke, "Estrogens, BRCA1, and breast cancer," Cancer Research, vol. 60, no. 18, pp. 4993-5001, 2000.

[29] M. S. Farvid, W. Y. Chen, B. A. Rosner, R. M. Tamimi, W. C. Willett, and A. H. Eliassen, "Fruit and vegetable consumption and breast cancer incidence: repeated measures over 30 years of follow-up," International Journal of Cancer, vol. 144, no. 7, pp. 1496-1510, 2019.

[30] S. Hurtado-Barroso, M. Trius-Soler, R. M. Lamuela-Raventós, and R. Zamora-Ros, "Vegetable and fruit consumption and prognosis among cancer survivors: a systematic review and meta-analysis of cohort studies," Advances in Nutrition, vol. 11, no. 6, pp. 1569-1582, 2020.

[31] J. He, Y. Gu, and S. Zhang, "Consumption of vegetables and fruits and breast cancer survival: a systematic review and metaanalysis," Scientific Reports, vol. 7, no. 1, pp. 599-610, 2017.

[32] C. Sánchez-Quesada, A. Romanos-Nanclares, A. M. Navarro, A. Gea, S. Cervantes, and M. Á Martínez-González, "Coffee consumption and breast cancer risk in the SUN project," European Journal of Nutrition, vol. 59, pp. 1-11, 2020.

[33] A. Lafranconi, A. Micek, P. De Paoli et al., "Coffee intake decreases risk of postmenopausal breast cancer: a dose-response meta-analysis on prospective cohort studies," Nutrients, vol. 10, no. 2, p. 112, 2018.

[34] A. Haraldsdottir, L. Steingrimsdottir, U. A. Valdimarsdottir et al., "Early life residence, fish consumption, and risk of breast cancer," Cancer Epidemiology Biomarkers \& Prevention, vol. 26, no. 3, pp. 346-354, 2017.

[35] J. Pérez-Jiménez, M. Elena Díaz-Rubio, and F. Saura-Calixto, "Contribution of macromolecular antioxidants to dietary antioxidant capacity: a Study in the Spanish Mediterranean Diet," Plant Foods for Human Nutrition, vol. 70, no. 4, pp. 365-370, 2015.

[36] L. M. León-Muñoz, P. Guallar-Castillón, A. Graciani et al., "Adherence to the Mediterranean diet pattern has declined in Spanish adults," Journal of Nutrition, vol. 142, no. 10, pp. 1843-1850, 2012.

[37] M. Iriti and E. M. Varoni, "Melatonin in Mediterranean diet, a new perspective," Journal of the Science of Food and Agriculture, vol. 95, no. 12, pp. 2355-2359, 2015.

[38] A. Abdal Dayem, H. Choi, G.-M. Yang, K. Kim, S. Saha, and S.-G. Cho, "The anti-cancer effect of polyphenols against breast cancer and cancer stem cells: molecular mechanisms," Nutrients, vol. 8, no. 9, p. 581, 2016.

[39] S. Judé, S. Roger, E. Martel et al., "Dietary long-chain omega-3 fatty acids of marine origin: a comparison of their protective effects on coronary heart disease and breast cancers," Progress in Biophysics and Molecular Biology, vol. 90, no. 1-3, pp. 299-325, 2006.

[40] N. F. Santos-Sánchez, R. Salas-Coronado, C. VillanuevaCañongo, and B. Hernández-Carlos, Antioxidant Compounds and Their Antioxidant Mechanism, IntechOpen London, London, UK, 2019.
[41] A. Mawson, A. Lai, J. S. Carroll, C. M. Sergio, C. J. Mitchell, and B. Sarcevic, "Estrogen and insulin/IGF-1 cooperatively stimulate cell cycle progression in MCF-7 breast cancer cells through differential regulation of c-Myc and cyclin D1," Molecular and Cellular Endocrinology, vol. 229, no. 1-2, pp. 161-173, 2005.

[42] S. M. Alavian, A. Esmaillzadeh, P. Adibi, and L. Azadbakht, "Dietary quality indices and biochemical parameters among patients with non alcoholic fatty liver disease (NAFLD)," Hepatitis Monthly, vol. 13, no. 7, 2013.

[43] M. S. Farvid, M. C. Stern, T. Norat et al., "Consumption of red and processed meat and breast cancer incidence: a systematic review and meta-analysis of prospective studies," International Journal of Cancer, vol. 143, no. 11, pp. 2787-2799, 2018.

[44] J. J. Anderson, N. D. M. Darwis, D. F. Mackay et al., "Red and processed meat consumption and breast cancer: UK Biobank cohort study and meta-analysis," European Journal of Cancer, vol. 90, pp. 73-82, 2018.

[45] A. N. Samraj, O. M. T. Pearce, H. Läubli et al., "A red meatderived glycan promotes inflammation and cancer progression," Proceedings of the National Academy of Sciences, vol. 112, no. 2, pp. 542-547, 2015.

[46] S. H. Ley, Q. Sun, W. C. Willett et al., “Associations between red meat intake and biomarkers of inflammation and glucose metabolism in women," The American Journal of Clinical Nutrition, vol. 99, no. 2, pp. 352-360, 2014.

[47] A. Ronco, E. De Stefani, M. Mendilaharsu, and H. DeneoPellegrini, "Meat, fat and risk of breast cancer: a case-control study from Uruguay," International Journal of Cancer, vol. 65, no. 3, pp. 328-331, 1996. 